\title{
ANÁLISE POSTURAL E DO MOVIMENTO DE OMBROS EM PACIENTES PÓS MASTECTOMIZADOS SOB INTERVENÇÃO FISIOTERÊUTICA
}

\author{
Walkíria Monteiro Alves', Barbara Benevides de Lima', Delcarla Rezende Batista', Laura \\ Boechat Pires de Almeida Sales ${ }^{1}$, Dib Chacur Abdalla ${ }^{2}$ \& Patrícia Junqueira Ferraz Baracat ${ }^{3 *}$
}

\section{RESUMO}

ALVES W.M.; LIMA, B.B.; BATISTA, D.R.; SALES, L.B.P.A.; ABDALLA, D.C. \& BARACAT, P.J.F. Análise postural e do movimento de ombros em pacientes pós mastectomizados sob intervenção fisioterêutica. Perspectivas Online: Biológicas e Saúde, v.7, n.24, p.1-13, 2017.

O câncer de mama é o segundo tipo de câncer mais frequente no mundo. Apesar do avanço das técnicas cirúrgicas para o tratamento, há forte probabilidade de complicações pós-operatórias cinético-funcionais. Avaliar a postura e o arco de movimento (ADM) do ombro de pacientes mastectomizados antes e após a intervenção fisioterapêutica. Estudo série de casos que acompanhou 7 pacientes submetidos à mastectomia. A avaliação postural e do ADM para abdução e rotação externa do ombro foi realizada através da biofotometria e análise de dados pelo software IMAGE J antes e após 10 sessões de tratamento fisioterapêutico. Os dados foram submetidos ao teste $\mathrm{T}$ com significância de 5\%.
Sete pacientes com idade de 56,8 $\pm 5,5$ anos, gênero feminino foram submetidos à intervenção e apresentaram ganho de arco para os movimentos de rotação externa $(\mathrm{p}=0,018)$ e abdução de ombro $(\mathrm{p}=0,003)$. $\mathrm{Na}$ análise postural houve maior simetria do nivelamento dos ângulos inferiores das escápulas $(p=0,03)$. A intervenção fisioterapêutica exerce influência sobre o ganho de arco de movimento e diminuição das disfunções posturais secundárias a mastectomia. Estudos com maior número de participantes e maior período de tratamento são de extrema importância para a elaboração de conclusões mais sólidas.

Palavra-chave: Câncer de mama, mastectomia, ADM, fisioterapia, postura. 


\begin{abstract}
Breast cancer is the second most common cancer in the world. Despite the advancement of surgical techniques for treatment there is a considerable probability of postoperative kinetic-functional complications. To evaluate the posture and shoulder movement of mastectomized patients before and after the physiotherapeutic intervention. A case series study was carried out with 7 patients submitted to mastectomy. The postural and ADM evaluation for abduction and external rotation of the shoulder was performed through biophotometry and data analysis by IMAGE J software before and after 10 sessions of physiotherapeutic treatment. Data were submitted

to the $\mathrm{T}$ test with significance of $5 \%$. Seven patients, $56.8 \pm 5.5$ years old, female, were submitted to physical therapy intervention, all of them presented an arc gain for the external rotation $(\mathrm{p}=0.018)$ and abduction of the shoulder $(\mathrm{P}=0.003)$. Posture analyses shows greater symmetry of the leveling of the lower scapula angles $(p=0.03)$. Physiotherapeutic intervention exerts influence on the movement gain and diminish postural dysfunctions secondary to mastectomy, studies with a larger number of participants and a longer period of treatment are extremely important for the elaboration of more solid conclusions.
\end{abstract}

Keywords: Breast cancer, mastectomy, WMD, physiotherapy, posture.

\footnotetext{
${ }^{1}$ Acadêmicos em Fisioterapia do ISECENSA e colaboradores do PROVIC - Rua Salvador Correa, 139, Centro, Campos dos Goytacazes, RJ, CEP: 28035-310, Brasil

${ }^{2}$ Professor de ginecologia e mastologia FMC/HEAA - Mestre em ginecologia UFRJ

${ }^{3}$ Osteopata DO, Mestre em Ciências da Reabilitação, professora do ISECENSA - Rua Salvador Correa, 139, Centro, Campos dos Goytacazes, RJ, CEP: 28035-310, Brasil

(*) e-mail: patbaracat@yahoo.com.br

Data de chegada: 17/04/2017 Aceito para publicação: 12/06/2017
} 


\section{INTRODUÇ̃̃̃O}

O câncer de mama é uma das neoplasias mais comuns entre as mulheres. Atualmente representa o segundo tipo de câncer mais incidente no sexo feminino, com raro acometimento no sexo masculino. Estimase de 57.960 novos casos de câncer de mama em 2016 no Brasil. Em 2013 foram contabilizadas 14.388 mortes sendo 181 homens e 14.206 mulheres. No entanto, é o tipo de neoplasia que apresenta uma considerável possibilidade de sobrevida quando diagnosticada precocemente. Os melhores meios para detecção precoce do câncer de mama são a mamografia e o exame clínico, mas a ultrassonografia também pode ser utilizada como recurso (INCA 2016; LIMA-COSTA et al., 2007; MINISTÉRIO DA SAÚDE, 2016).

Devido às recentes evoluções terapêuticas e diagnósticas, o câncer vai deixando de ser uma doença repetidamente fatal e atribuindo características de uma doença crônica, que deverá ser controlada em longo prazo. O tratamento varia de acordo com o estágio da doença, podendo ser local como a cirurgia e radioterapia e sistêmico como a quimioterapia e terapia biológica. A técnica de dissecção axilar, conservadora ou radical, ocorre após o tratamento cirúrgico do câncer de mama. Tal procedimento, frequentemente associado a radioterapia pós-operatória, pode causar severas complicações para o membro acometido à cirurgia. Hemorragias, alterações de sensibilidade, comprometimento da capacidade respiratória, lesões no plexo braquial, dor, diminuição da força muscular, fibrose áxilo-peitoral, redução ou perda da amplitude de movimento e o linfedema do braço homolateral são consideradas consequências do tratamento do câncer de mama (FERREIRA et al., 2010; INCA, 2016; BATISTON et al., 2005; LAHOZ et al., 2010).

No que concerne à postura corporal, o deslocamento do centro de gravidade e as modificações de um segmento do corpo são complicações que podem acarretar instabilidade e alteração do equilíbrio. As consequências do tratamento e a ausência do peso da mama contribuem negativamente na biomecânica corporal das pacientes e podem gerar deformações irreversíveis se não forem tratadas a tempo. As alterações posturais mais comuns nas mulheres em intervenção pelo câncer de mama são anteriorização e inclinação da cabeça para o lado contralateral à cirurgia, protrusão e elevação de ombro, alteração nos ângulos de cifose e lordose lombar e torácica, escápulas retraídas e rotação da pelve (PEREZ, 2015; ROSTKOWSKA et al., 2006; CIESLA et al., 2010; MALICKA et al., 2010).

Segundo Pereira et al. (2005) a fisioterapia no pós-operatório imediato é importante na prevenção de possíveis adversidades provenientes da dissecção axilar no tratamento da neoplasia mamária. Quando introduzidos exercícios cinesioterápicos, logo nos primeiros dias posteriores a cirurgia, é possível evitar principalmente o linfedema, as retrações miotendíneas, possibilitando o retorno mais breve às atividades funcionais e de vida diária. A partir de 1970 observou-se a necessidade de melhor qualidade de vida para pacientes mastectomizadas. A fisioterapia atua também no fornecimento de informações e suporte ao paciente, auxiliando nos cuidados pré e pós-operatórios (SILVA, 2002; ABREU et al., 2002).

A partir das considerações acima, o objetivo deste estudo foi diagnosticar as principais alterações posturais em pacientes mastectomizados e quantificar o arco de movimento de abdução e rotação externa do ombro homolateral à cirurgia antes e após intervenção fisioterapêutica, interferindo assim no surgimento ou agravamento de possíveis complicações. 


\section{METODOLOGIA}

Foi realizado um estudo série de casos prospectivo com 7 (sete) pacientes com câncer de mama, de pós-operatório de mastectomia, idade entre 40 e 75 anos e índice de massa corpórea (IMC) entre 18,5 e 32,3 $\mathrm{kg} / \mathrm{m}^{2}$ que apresentassem diminuição do arco de movimento do ombro homolateral a cirurgia para os movimentos de abdução e rotação externa. Não foram incluídos no estudo pacientes com histórico de lesão ortopédica importante, ferida no tórax ou no braço homolateral à intervenção cirúrgica, lesão de nervos periféricos, déficit de cognição, mastectomia bilateral e reconstrução mamária. Foram excluídos do estudo pacientes que não completaram o tempo de tratamento e as sessões propostas na metodologia. $\mathrm{O}$ estudo foi aprovado pelo Comitê de Ética em Pesquisa dos Institutos Superiores de Ensino do CENSA, de acordo com a resolução 466/12 e todos os indivíduos assinaram o termo de consentimento livre e esclarecido.

Todos os voluntários foram encaminhados pelo serviço de ginecologia e mastologia do Hospital Escola Álvaro Alvim e orientados sobre os objetivos do tratamento que seria realizado. Após concordarem, assinaram o Termo de Consentimento Livre e Esclarecido. Foi feita uma ficha de identificação com dados pessoais e dados sobre a intervenção cirúrgica de cada paciente como tipo de cirurgia e tempo de realização da mesma. Os pacientes deveriam ter no mínimo 20 dias de pós operatório e o tempo máximo não foi determinado sendo o principal critério de inclusão a ocorrência de hipomobilidade articular para os movimentos propostos no estudo. Para determinar ou não a presença de linfedema, considerou-se a diferença mínima de $2 \mathrm{~cm}$ na circunferência entre as medidas dos dois membros superiores. Verificou-se 8 medidas, sendo o ponto de partida: prega do cotovelo, 5,10 e $15 \mathrm{~cm}$ acima e 5,10 e $15 \mathrm{~cm}$ abaixo e o dorso da mão.

As imagens foram captadas através da câmera fotográfica digital (Sony Cyber-shot 16.2 megapixels), posicionada sobre um tripé alinhado a $1 \mathrm{~m}$ do chão, com as participantes com vestuário adequado a uma avaliação postural, localizadas sobre uma marca desenhada no chão, com os pés equidistantes. Foram feitas avaliações de postura e de amplitude de movimento de braço (abdução e rotação externa) nesses pacientes por meio do programa Image $J$, versão 2013, que corresponde a um software para análise das imagens que permite a mensuração e análise da variação angular do movimento de cada participante.

Os pacientes foram avaliados em dois momentos: antes do protocolo de intervenção (PRE) e após 5 semanas de tratamento (POS). A amplitude de movimento dos membros superiores contou com os movimentos de abdução e rotação externa. A análise das imagens dos movimentos e postura foi realizada individualmente. Foi realizado o mapeamento com o auxílio de marcadores autoadesivos, de coloração cinza, que serviram de guia para o processamento da imagem e traçado dos ângulos analisados. A vista anterior considerou o acrômio como ponto de referência, foram analisados principalmente o alinhamento horizontal da cabeça e dos ombros. Na vista posterior, foram demarcados os seguintes pontos: processos espinhosos da segunda vértebra torácica (T2), da sexta vértebra torácica (T6), da oitava vértebra torácica (T8), ângulo superior e inferior da escápula, superfície triangular da escápula, base do acrômio. Nessa vista, foram avaliados a simetria horizontal da escápula relacionada a T2 e eixo vertebral. As vistas laterais direita ou esquerda apresentaram as seguintes demarcações: articulação temporomandibular (ATM), acrômio, processo espinhoso da segunda vértebra torácica (T2). Nesta postura foi considerada o alinhamento horizontal da cabeça relacionado a $\mathrm{T} 2$.

Persp. online: biol. \& saúde, Campos dos Goytacazes, 24 (7), 1-13, 2017

seer.perspectivasonline.com.br 
Figura 1- Demarcação dos pontos anatômicos na vista posterior.

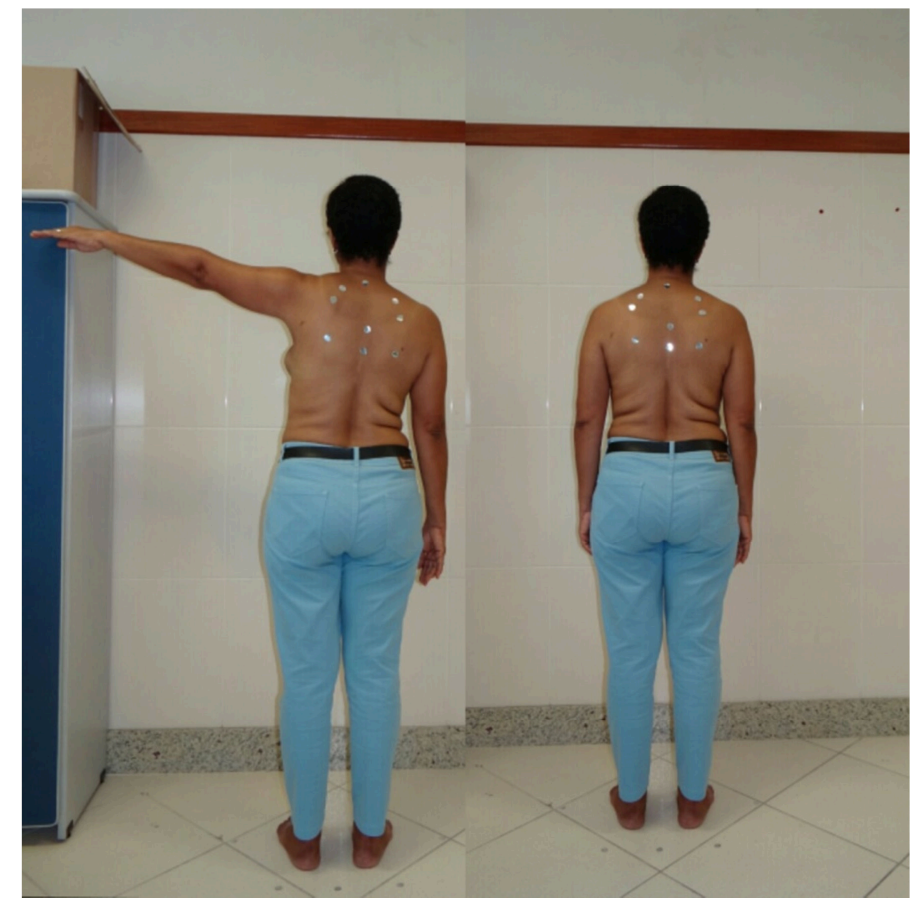

*As imagens do movimento de abdução foram captadas bilateralmente, para posterior comparação.

Todos os pacientes foram submetidos a um protocolo de tratamento de 5 (cinco) semanas, sendo 2 (duas) sessões por semana, totalizando 10 (dez) sessões. Com duração em média de 50 (cinquenta) minutos. Realizado assim o seguinte protocolo:

1- Alongamento de forma ativa para músculos trapézio, peitoral e fibras anteriores de deltóide e passivo para ECOM e escalenos.

2- Técnicas de descolamento e liberação cicatricial.

3- Técnica de ganho de amplitude através da liberação da musculatura adutora do braço.

4- Mobilização de escápula.

5- Foram orientados a 2 auto-posturas de RPG rã no chão, com braços abertos e rã no ar com braços fechados com duração de 10 minutos cada, totalizando 20 minutos.

Pacientes foram orientados a prática de exercícios domiciliares composta por 10 repetições a saber: inclinação e rotação cervicais, flexão e extensão de ombros, cotovelos, punhos e dedos; adução e abdução horizontal de ombros, movimentos de flexão de braço e antebraço com o auxílio de bastões, elevação e depressão de escápulas, finalizando com os alongamentos propostos na conduta descrita (1). Os pacientes foram orientados sobre cada exercício e caso sentissem desconforto ou alguma desestabilização ventilatória ou pressórica eram realizados exercícios diafragmáticos a fim de contribuir para a estabilização do quadro.

Os dados obtidos foram inseridos e organizados em planilhas do programa Microsoft Excel ${ }^{\circledR} 2016$ (Microsoft Corporation; Redmond, WA, EUA), possibilitando o cálculo de média e desvio padrão para cada variável. Para análise dos resultados e confecção dos gráficos foi utilizado o mesmo programa Microsoft Excel $^{\circledR} 2016$ (Microsoft Corporation; Redmond, WA, EUA). Para a análise dos domínios sobre a análise postural e ADM do ombro foi utilizado o teste-t pareado. Foi utilizado um nível de significância de 5\%. 


\section{RESULTADOS}

O presente estudo iniciou-se com 9 pacientes com idade entre 49 e 66 anos, com média de 56,8 5,5 anos, apresentaram peso de $67,1 \pm 7,9 \mathrm{~kg}$, sendo $85,7 \%$ do gênero feminino. Destes, 2 participantes foram excluídos por apresentarem assiduidade menor que $75 \%$, sendo 1 destes o único do gênero masculino participante. Os tratamentos cirúrgicos pelo qual os pacientes foram submetidos para retirada do câncer de mama não foram conservadores em apenas $14,2 \%$ dos casos. A mastectomia à direita teve prevalência de $57,1 \%$, nenhum dos participantes apresentou histórico relacionado ao tabagismo ou ao etilismo; $71,4 \%$ relataram haver casos de câncer na família e não houve nenhuma apresentação de linfedema. As características da amostra estão representadas na Tabela 1. Em relação à frequência de dor na coluna antes da cirurgia, $42,8 \%$ dos pacientes responderam sim, e ao final da coleta todos relataram remissão da dor.

Tabela 1: Características da amostra e resposta do questionário de identificação.

\begin{tabular}{ccccc}
\hline Paciente & Idade & Sedentarismo & HF & Mastectomia \\
\hline $\mathbf{1}$ & 49 & Sim & Sim & Total E \\
$\mathbf{2}$ & 66 & Sim & Sim & Parcial D \\
$\mathbf{4}$ & 58 & Sim & Sim & Total D \\
$\mathbf{5}$ & 54 & Sim & Sim & Total D \\
$\mathbf{6}$ & 53 & Sim & Não & Total E \\
7 & 55 & Sim & Sim & Total E \\
Média \pm DP & 56,8 & Não & Não & Total D \\
& & $6 / 7(85 \%)$ & $5 / 7(71 \%)$ & $4 / 7(57 \%)$ D \\
\hline
\end{tabular}

HF: histórico familiar

A tabela 2 corresponde a análise da amplitude de movimento abdução de ombro no pré e pós tratamento $\left(97,4 \pm 32,9^{\circ}\right.$ vs $\left.173,7 \pm 33,5^{\circ} ; p=0,003\right)$, conforme demonstrado na figura 7 . O mesmo resultado foi observado para rotação externa $\left(38,2 \pm 21,5^{\circ}\right.$ vs $\left.63,2 \pm 17,1^{\circ} ; p=0,018\right)$, este apresentado na figura 8 . Ambos os resultados obtiveram diferença estatisticamente significativa.

Tabela 2: Amplitude de movimento (ADM) do membro superior PRÉ e PÓS do tratamento e p-valor.

\begin{tabular}{lccc}
\hline ADM Ativa & PRÉ & PÓS & P-VALOR \\
\hline Abdução & $97,4 \pm 32,9$ & $173,7 \pm 33,5$ & $0,003^{*}$ \\
RE & $38,2 \pm 21,5$ & $63,2 \pm 17,1$ & $0,018^{*}$ \\
\hline
\end{tabular}

ADM: amplitude de movimento; $R E$ : rotação externa.

*Diferença estatisticamente significativa $(p<0,05)$. 


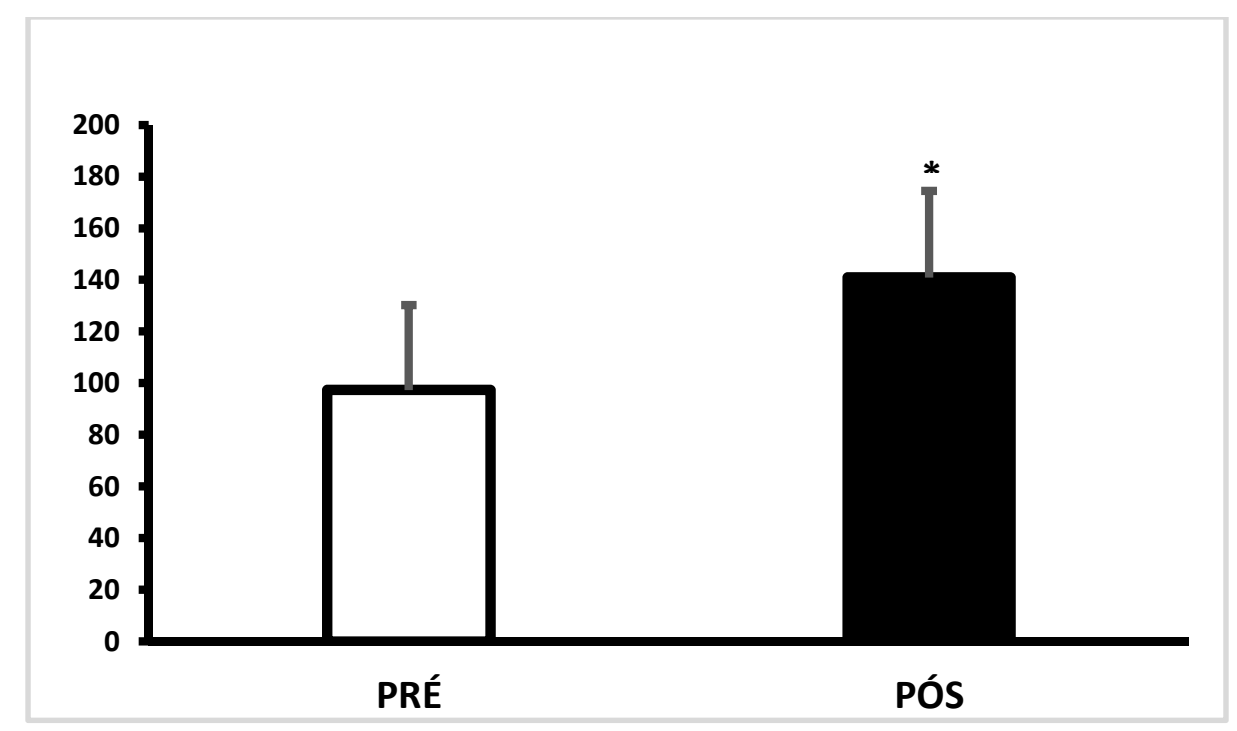

Figura 2: Média \pm Desvio padrão da amplitude de movimento (ADM) para abdução de ombro, nos instantes pré (branco) e pós (preto). *Diferenças estatisticamente significativa em relação ao PRÉ $(\mathrm{p}<0,05)$.

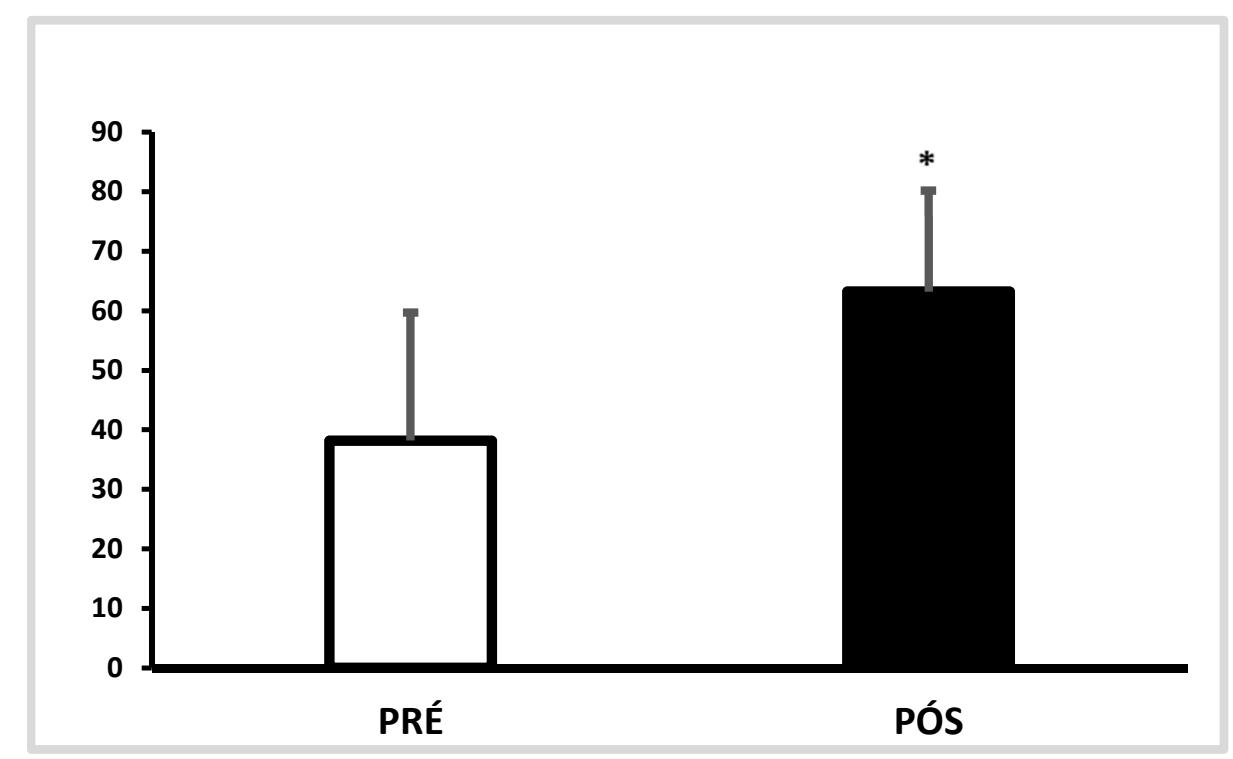

Figura 3: Média \pm Desvio padrão da amplitude de movimento (ADM) para rotação externa de ombro, nos instantes pré (branco) e pós (preto). Foi observado. *Diferenças estatisticamente significativa em relação ao PRÉ ( $\mathrm{p}<0,05)$. 
A figura 4 corresponde a análise da flexão anterior de tronco no pré e pós intervenção $\left(2,1 \pm 0,8^{\circ} v s\right.$ $\left.1,5 \pm 0,8^{\circ} ; \mathrm{p}=0,08\right)$.

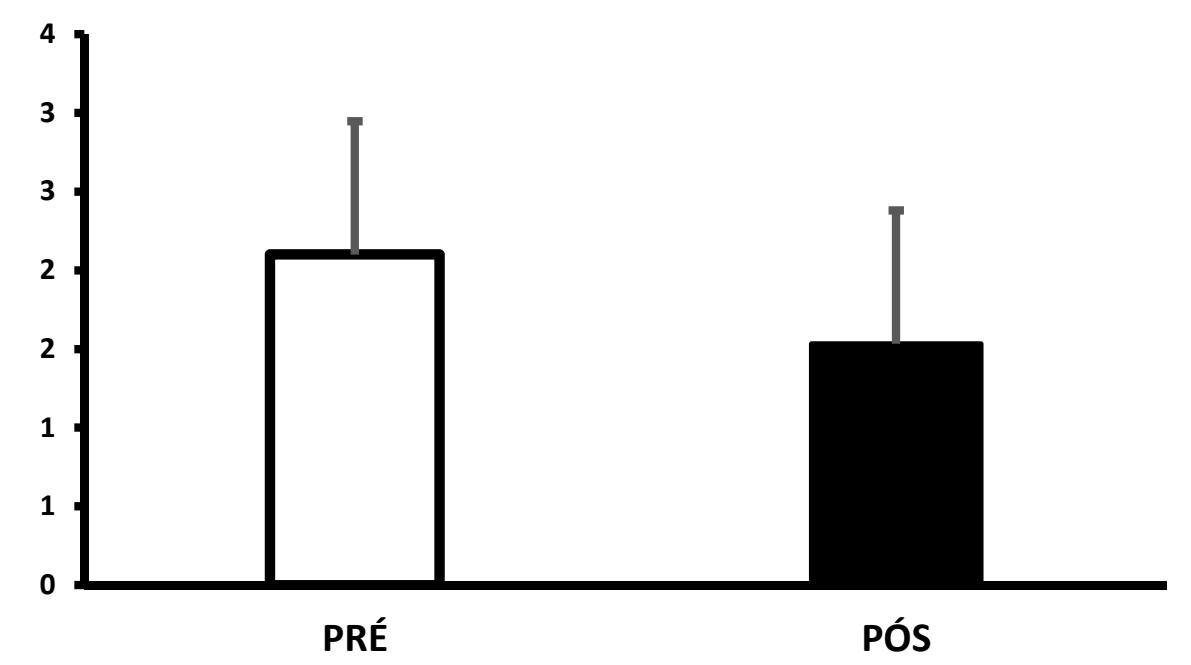

Figura 4: Média \pm Desvio padrão da análise postural para inclinação de tronco, nos instantes pré (branco) e pós (preto). Não foram observadas diferenças estatisticamente significativas entre os momentos PRÉ e PÓS $(\mathrm{p}>0,05)$.

A figura 5 corresponde a análise da anteriorização de cabeça no pré e pós intervenção $\left(33,2 \pm 3,6^{\circ} v S\right.$ $\left.32,2 \pm 1,8^{\circ} ; \mathrm{p}=0,27\right)$. Observa-se diminuição do desvio padrão na comparação dos resultados.

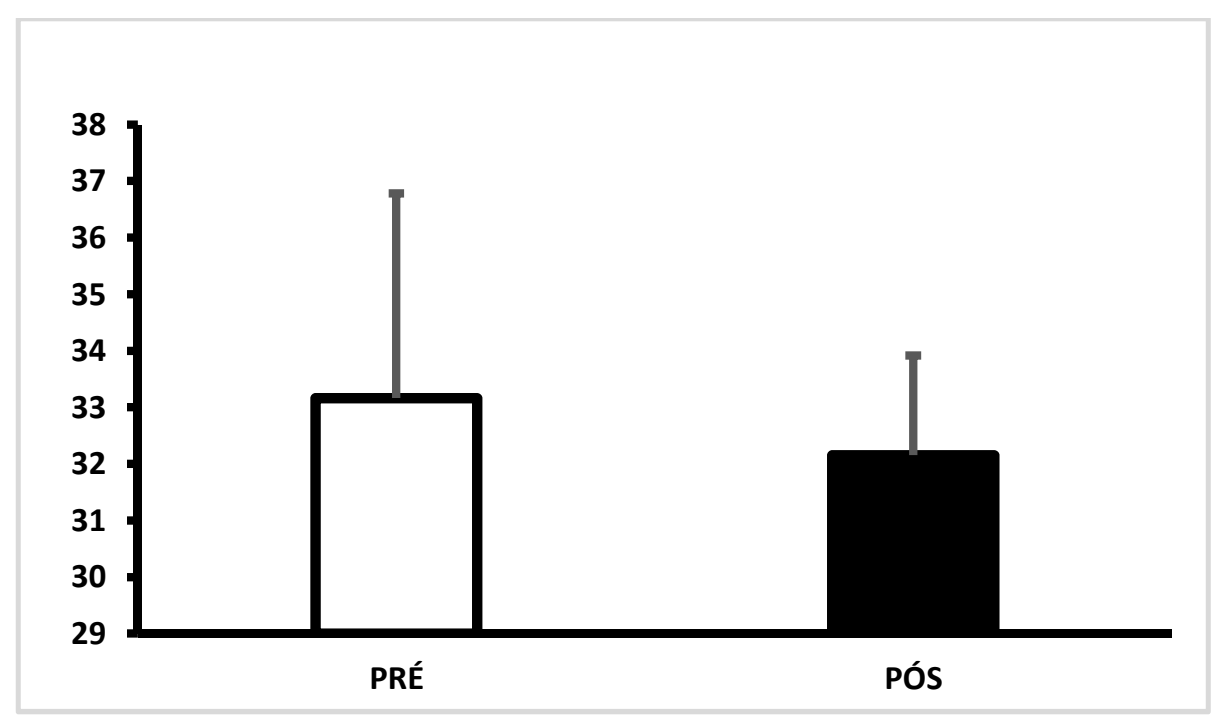

Figura 5: Média \pm Desvio padrão da análise postural para anteriorização de cabeça, nos instantes pré (branco) e pós (preto). Não foram observadas diferenças estatisticamente significativas entre os momentos PRÉ e PÓS ( $\mathrm{p}>0,05)$. 
A figura 6 corresponde a análise do nivelamento do ângulo inferior da escápula no período pré e pós intervenção $\left(4,8 \pm 2,2^{\circ}\right.$ vs $\left.2,7 \pm 1,4^{\circ} ; \mathrm{p}=0,03\right)$.

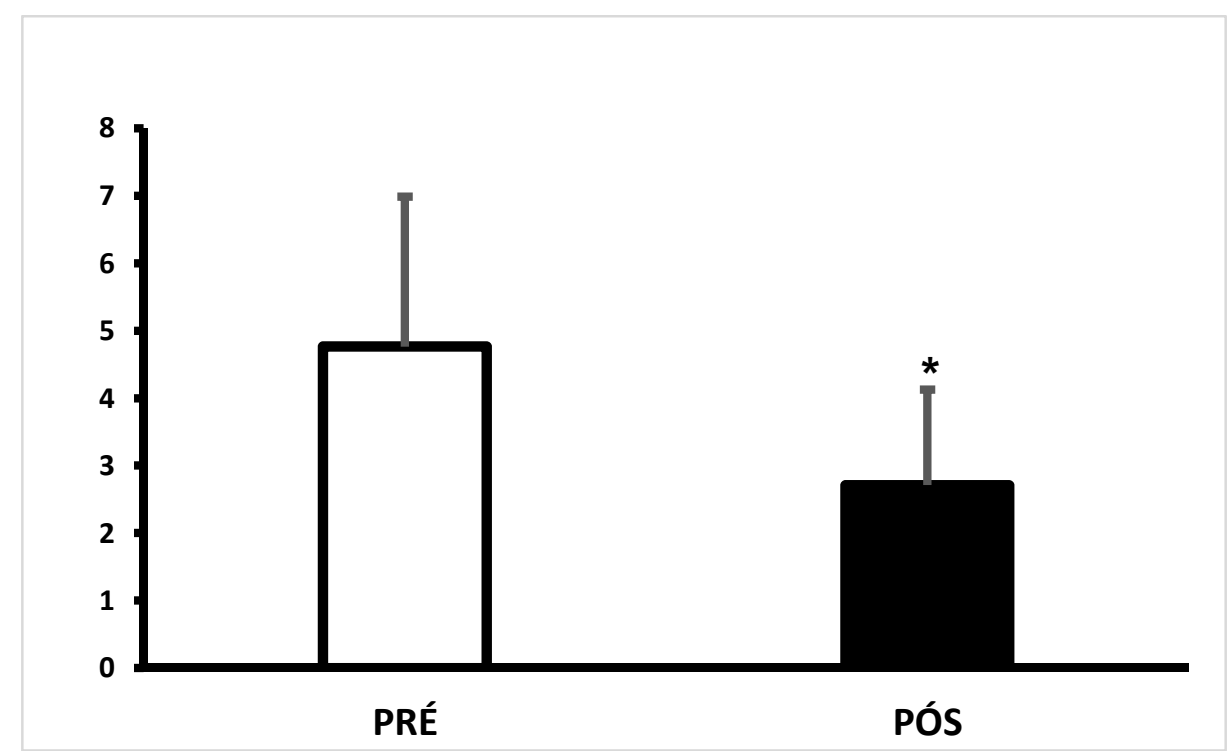

Figura 6: Média \pm Desvio padrão da análise postural para nivelamento do ângulo inferior da escápula, nos instantes pré (branco) e pós (preto). *Diferença estatisticamente significativa em relação ao PRÉ $(\mathrm{p}<0,05)$.

A figura 7 corresponde a análise da simetria angular dos acrômios no pré e pós intervenção $\left(1,3 \pm 1,3^{\circ}\right.$ vs $\left.1,9 \pm 2,3^{\circ} ; \mathrm{p}=0,10\right)$.

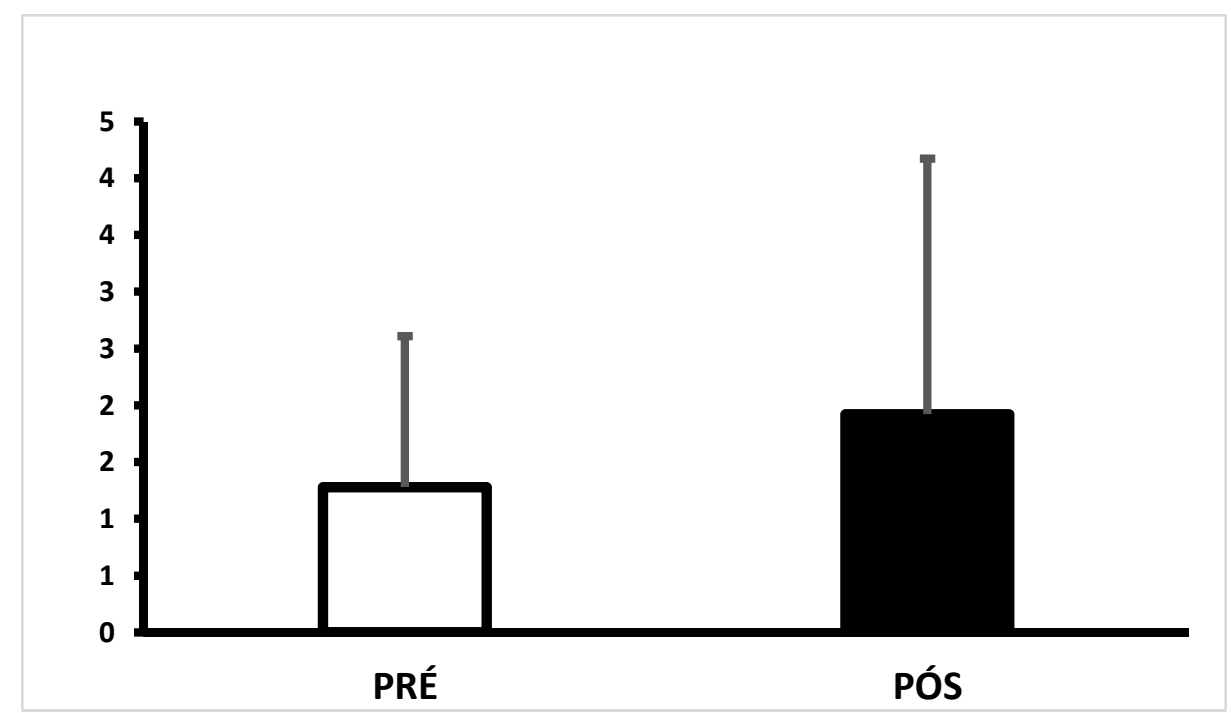

Figura 7: Média \pm Desvio padrão da análise postural para simetria da altura dos acrômios, nos instantes pré (branco) e pós (preto). Não foram observadas diferenças estatisticamente significativas entre os momentos PRÉ e PÓS ( $p>0,05)$. 


\section{DISCUSSÃO}

A mastectomia radical modificada é uma cirurgia invasiva que gera uma redução da amplitude do movimento do membro superior, podendo comprometer também a força muscular dessa mesma região. Batiston e Santiago (2005) observaram que $68,8 \%$ das pacientes foram submetidas ao tratamento cirúrgico radical. A mastectomia radical também foi o procedimento mais utilizado neste estudo sendo $85,7 \%$ dos procedimentos realizados. Quando são necessários procedimentos de amplo porte para a retirada da mama, especialmente quando há esvaziamento axilar, em 63,6\% dos casos a paciente pode apresentar diversas complicações (SASAKI E LAMARI, 1997).

O câncer de mama em homens é uma doença atípica e pouco conhecida. Corresponde a $1 \%$ em comparação a todas as neoplasias de mama. Anatomicamente a mama masculina é simular a um atraso da feminina. A ocorrência varia de país a país, dos recém-diagnosticados de câncer de mama em homens há cerca de 1,2\% nos Estados Unidos e 1\% na Europa enquanto na África sub-saariana tem um percentual elevado, cerca de 15\% de todos os cânceres (HAAS et al., 2009). O câncer de mama em homens e mulheres é semelhante, mas os casos masculinos exibem particularidades imuno-histoquímicas (NOGUEIRA et al, 2014). Esse estudo contou inicialmente com um paciente do sexo masculino e oito do sexo feminino confirmando a maior incidência da patologia nas mulheres. Até o desfecho 2 pacientes foram excluídos por não realizarem todo o tratamento proposto, entre eles o único participante masculino.

Quando há necessidade de retirada dos gânglios linfáticos visando reduzir a ocorrência de metástase, a magnitude da cirurgia pode ser motivo de desconforto e limitação biomecânica para os movimentos do ombro homolateral ao procedimento (SILVA et al., 2014). A radioterapia é utilizada tanto na fase préoperatória quanto na fase pós-operatória, com o objetivo de diminuir o tamanho do tumor antes da intervenção cirúrgica e destruir as células neoplásicas após a cirurgia (SCHETTINO E CASSALI, 2010). Neste estudo cerca de $42,8 \%$ dos pacientes realizaram radioterapia antes da intervenção cirúrgica. Nesvold et al. (2008), realizou um estudo comparativo entre mastectomia radical modificada (RM) e o tratamento conservador (TC) associado à radioterapia, revelando que $24 \%$ das mulheres submetidas à RM possuíam restrição ao movimento de flexão de ombro em oposição a 7\% das submetidas a TC. Os pacientes participantes deste estudo foram submetidos, em sua maioria a mastectomia total, o que justificam os dados da avaliação. Para a realização da Tomografia Computadorizada que possibilita a marcação da área a ser irradiada e também para a realização do procedimento de radioterapia, se faz necessário que o paciente mantenha o membro superior homolateral em posição de $90^{\circ}$ de abdução e rotação externa a fim de manter a área a ser tratada exposta. Não raro, pacientes apresentam restrições importantes para estes movimentos que chegam a prejudicar e até retardar o início da radioterapia.

A afirmação acima se apoia nos achados de vários autores. Lahoz et al. (2010) observaram que grande parte das mulheres apresentavam limitação da $\mathrm{ADM}$ do ombro homolateral à cirurgia, o que prejudicava a execução dos movimentos de flexão, rotação externa e abdução. Bregagnol e Dias (2009) também destacaram a diminuição do ADM do ombro para os mesmos movimentos 30 dias após a cirurgia. Para Silva et al. (2004) qualquer limitação acima de $30^{\circ}$, tanto para flexão quanto para abdução, torna-se incompatível com a realização das tarefas básicas diárias. Os resultados deste estudo concordam com os anteriores citados, os pacientes avaliados apresentaram inicialmente redução do ADM para os movimentos de abdução e rotação externa. Na avaliação pós-intervenção foi verificada a redução de aderência articular e periarticular geradoras de hipomobilidade e dor, restaurando assim a funcionalidade para os movimentos avaliados de todos os participantes $(\mathrm{p}<0,05)$.

A manutenção da postura corporal é uma missão complexa, pois envolve a interação de estruturas proprioceptivas e exteroceptivas do sistema neural e musculoesquelético. Após a mastectomia, os pacientes tendem a adotar posturas antálgicas em decorrência da dor, restringindo o esforço e dificultando a mobilização ativa. O paciente desorganiza a sua biomecânica corporal na tentativa de se reequilibrar,

Persp. online: biol. \& saúde, Campos dos Goytacazes, 24 (7), 1-13, 2017

seer.perspectivasonline.com.br 
apresentando frequentemente contratura na região cervical e cintura escapular, realizando elevação do ombro e abdução da escápula homolateral à cirurgia (CIESTA E POLOM, 2010).

Malicka et al. (2010) através da fotogrametria, avaliaram e compararam as posturas de mulheres após mastectomia e mulheres saudáveis, observando que $82,3 \%$ das mulheres após o tratamento apresentavam disfunções posturais em oposição a 35,1\% das saudáveis. Rostkowska, Bak e Samborski (2006) avaliaram e compararam a postura corporal de mulheres saudáveis e mulheres após câncer de mama e concluíram que pacientes mastectomizadas apresentavam mais desvios posturais, a saber, maior ângulo de inclinação anterior e lateral do tronco, maior valor dos ângulos das curvaturas vertebrais e ombros e escápulas assimétricas mesmo após três anos da cirurgia. Porém a inclinação do tronco não apresentou correlação com o lado da cirurgia, mas sim, com a idade. Neste estudo, os pacientes relataram desconforto e dor após a cirurgia, levando a postura antálgica de enrolamento horizontal dos ombros.

Os pacientes que foram submetidos à mastectomia à esquerda apresentaram o ombro esquerdo mais elevado quando comparados aos que realizaram a cirurgia do lado direito. Ciesta e Polom (2010) relataram que seis meses após a mastectomia, as mulheres apresentaram elevação e protrusão do ombro associados à rotação escapular homolateral à cirurgia. Camargo e Marx (2000) concluíram que tal ocorrência foi devido à falta de peso da mama que faz com que o ombro homolateral a cirurgia se eleve e gire internamente, abduzindo a escápula e gerando uma contratura muscular da região cervical e, como consequência, a dor. Corroborando o presente estudo, Rostkowska et al (2006) detectou a diferença na altura das escápulas em mulheres saudáveis, quando comparadas a mulheres mastectomizadas, que apresentavam escápulas mais elevadas. Todos os pacientes avaliados neste estudo apresentaram alterações posturais que foram minimizadas após as intervenções, principalmente no que diz respeito às variáveis de simetria dos ângulos da escápula, aspectos diretamente relacionados ao ganho de mobilidade do ombro.

Para Ciesta e Polom (2010), não há existência de alterações de fatores mecânicos pela retirada da mama e sim, alterações decorrentes de fatores psicológicos, dor e sensação de mutilação. Rostkowska et al. (2006) concluíram que após a mastectomia, foram adquiridas modificações significantes na postura, causadas pela limitação dos movimentos, além da circunstância dolorosa da coluna vertebral. Buscou-se aqui utilizar um método de avaliação e quantificação das possíveis alterações posturais e déficit da amplitude dos movimentos, a fim de compará-los nos momentos pré e pós-intervenção. Os resultados mostraram maior simetria para os ângulos posturais correspondentes ao nivelamento das escápulas em todos os pacientes após a intervenção fisioterapêutica. Porém em face aos resultados relacionados à inclinação de tronco, simetria na altura dos acrômios e anteriorização da cabeça, não se observou significância estatística $(p>0,05)$, sugerindo que as auto posturas de RPG não foram suficientes para melhora da disfunção postural.

A anteriorização da cabeça foi uma disfunção postural comum a todos os pacientes, concordando assim com o estudo de Roswtkowska et al. (2006) que constatou que mulheres operadas recentemente apresentavam mais anteriorização de tronco quando comparadas as que realizaram o procedimento há mais tempo, associando a adoção da postura anteriorizada ao conforto, alívio e proteção. Rossi et al. (2011) constatou que os exercícios de alongamento dos músculos e fáscias da cadeia anterior, tais como o esternocleidomastoideo (ECOM), realizados através das auto posturas de RPG contribuem para uma postura equilibrada e adequada, o que não foi observado nesta pesquisa. Este resultado pode ser atribuído ao curto período de tratamento proposto para minimizar disfunções tão importantes em pacientes que ainda estão em tratamento de câncer, patologia que gera, na maioria dos casos, instabilidade emocional e medo.

\section{CONCLUSÃO}

Este estudo teve como objetivo identificar alterações e limitações, apresentadas por pacientes mastectomizados e tratá-los através da terapia manual e auto posturas de RPG. Conclui-se que a intervenção fisioterapêutica exerceu influência sobre o alinhamento do corpo e na recuperação do ADM de rotação

Persp. online: biol. \& saúde, Campos dos Goytacazes, 24 (7), 1-13, 2017

seer.perspectivasonline.com.br 
externa e abdução do ombro, influenciando positivamente o conforto e a funcionalidade no pós-operatório de pacientes mastectomizados. Entretanto estudos com maior número de pacientes e com maior período de tratamento são necessários para conclusões mais sólidas sobre o efeito de auto posturas de reeducação postural global em pacientes submetidos a mastectomia.

\section{REFERÊNCIAS}

BATISTON, A. P.; SANTIAGO, S. M. Fisioterapia e complicações após tratamento cirúrgico do câncer de mama. Fisioterapia e pesquisa, v.12, n. 3, p. 30-35, 2005.

CIESTA, S.; POLOM, K. The effect immediate breast reconstruction with Becker-25 prosthesis on the preservation of proper body posture in patients after mastectomy. Eur J Surg Oncol, v. 36, n. 7, p. 625-631, 2010.

FERREIRA, N. M. L.; DUPAS, G.; COSTA, D. B.; SANCHEZ, K. O. L. Câncer e família: compreendendo os significados simbólicos. Cienc Cuid Saúde, v. 9, n. 2, p. 269-277, 2010.

HAAS, P.; COSTA, A. B.; SOUZA, A. P. Epidemiologia do câncer de mama em homens. Rev Inst Adolfo Lutz, v. 68 , n.3, p.476-81,2009.

INCA. Programa Nacional de Controle do Câncer de Mama, Rio de Janeiro, Brasil, 2016.

LAHOZ, M. A.; NYSSEN, S. M.; CORREIA, G. N.; GARCIA, A. P. U.; DRIUSSO, P. Qualidade funcional e qualidade de vida em mulheres pós-mastectomizadas. Revista Brasileira de Cancerologia, v. 56, n. 4, p. 423-430, 2010.

LIMA-COSTA, M. F.; MATOS, D. L. Prevalência e fatores associados à realização da mamografia na faixa etária de 50-69 anos: um estudo baseado na Pesquisa Nacional por Amostra de Domicílios. Cad. Saúde Pública, v. 23, n. 7, p. 1665-1673, 2007.

MALICKA, I.; BARCZYYK, K.; HANUSZKIEWICZ, J.; SKOLIMOWSKA, B.; WOZ 'NIEWSKI, M. Body posture of women after breast cancer treatment. Ortop Traumatol Rehabil. v. 12, n. 4, p. 353-361, 2010.

MINISTÉRIO DA SAÚDE (BR). Instituto Nacional de Câncer. Estimativa 2010: incidência de câncer no Brasil. Rio de Janeiro (RJ): INCA; 2009.

NOGUEIRA, S. P.; MENDONÇA, J. V.; PASQUALETTE, H. A. P. Câncer de mama em homens. Rev Bras Mastologia, n.24, v.4, p.109-114, 2014.

PEREIRA, C. M. A.; VIEIRA, E. O. R. Y.; ALCÂNTARA, P. S. M. Avaliação de protocolo de fisioterapia aplicado a pacientes mastectomizadas a Madden. Revista Brasileira de Oncologia, v. 51, n. 2, p. 143-148, 2005.

PEREZ C. S. Influência da mastectomia unilateral no equilíbrio estático e na marcha. 2015. 1p. Dissertação (pós-graduação) - Faculdade de Medicina de Ribeirão Preto, Universidade de São Paulo, São Paulo, 2015.

ROSTKOWSKA E.; BAK M.; SAMBORSKI W. Body posture in women after mastectomy and its changes as a result of rehabilitation. Adv Med Sci., v. 51, p. 287-97, 2006.

SCHETTINO, L. M. G. N.; CASSALI, G. D. Função pulmonar em mulheres com câncer de mama submetidas à radioterapia: um estudo piloto. Fisioterapia e Pesquisa, São Paulo, v.17, n.3, p.248-52, 2010. 
SASAKI, T.; LAMARI, N. M. Reabilitação funcional precoce pós-mastectomia. HB científica, v. 4, n.2, p. 121-127, 1997.

SILVA, E. C. S.; SILVA, J. M.; SILVA, L. F.; BATISTA, R. F.; SAMPAIO S.; CARNEIRO, P. F. P. Câncer de mama e qualidade de vida durante o tratamento radioterápico. Ciências biológicas e da saúde. v.1, n.3, p. 85-93, 2014.

SILVA, M. P. P. Efeitos da fisioterapia na recuperação e complicações no pós-operatório por câncer de mama: exercícios limitados versus não-limitados. 2002. Dissertação - Faculdade de Ciências Médicas, Universidade Estadual de Campinas, São Paulo, 2002. 